\title{
Multi-Period Technician Scheduling with Experience-based Service Times and Stochastic Customers
}

\author{
Xi Chen ${ }^{\mathrm{a}, \mathrm{c}}$, Barrett W. Thomas ${ }^{\mathrm{a}, *}$, Mike Hewitt $^{\mathrm{b}}$ \\ ${ }^{a}$ Department of Management Sciences, Tippie College of Business, University of Iowa, \\ Iowa City, IA, 52242 \\ ${ }^{b}$ Information Systems and Operations Management, Quinlan School of Business, Loyola \\ University Chicago, Chicago, IL, 60611 \\ ${ }^{c}$ Department of Management Science and Engineering, International Business School, \\ Beijing Foreign Studies University, Beijing, 100089, China
}

\begin{abstract}
This paper introduces the multi-period technician scheduling problem with experience-based service times and stochastic customers. In the problem, a manager must assign tasks of different types that are revealed at the start of each day to technicians who must complete the tasks that same day. As a technician gains experience with a type of task, the time that it takes to serve future tasks of that type is reduced (often referred to as experiential learning). As such, while the problem could be modeled as a single-period problem (i.e. focusing solely on the current day's tasks), we instead choose to model it as a multi-period problem and thus capture that daily decisions should recognize the long-term effects of learning. Specifically, we model the problem as a Markov decision process and introduce an approximate dynamic programming-based solution approach. The model can be adapted to handle cases of worker attrition and new task types. The solution approach relies on an approximation of the cost-to-go that uses forecasts of the next day's assignments for each technician and the resulting estimated time it will take to service those assignments given current period decisions. Using an extensive computational study, we demon-
\end{abstract}

\footnotetext{
${ }^{*}$ Corresponding author

Email address: barrett-thomas@uiowa.edu (Barrett W. Thomas)
}

Preprint submitted to Computers \& Operations Research

November 14, 2016

(C) 2016. This manuscript version is made available under the Elsevier user license http://www.elsevier.com/open-access/userlicense/1.0/ 
strate the value of our approach versus a myopic solution approach that views the problem as a single-period problem.

Keywords: Dynamic Programming, Technician Scheduling, Multi-period, Learning

2010 MSC: 00-01, 99-00

\section{Introduction}

As the global economy recovers, there is growing pressure in the skilled labour markets. According to the Hays Global Skills Index 2014, a statisticsbased study designed to assess the dynamics of skilled labour markets across

531 countries, this pressure continues to rise, particularly in economies that are returning to pre-crisis levels such as United States, Germany, and the United Kingdom [1]. Maintaining growth in a pressured labor market requires that companies use their expensive and limited labor resources to their greatest potential. One opportunity is for companies to take advantage of the capacity that is gained as employees learn by experience. Matching the right employee with the right job cannot only help a company meet its current needs, but also build capacity for meeting future demand growth as well as build the flexibility needed to buffer against demand uncertainty. Further, the ability to account for each individual employee's ability to learn allows companies to best deploy workers in the face of strategic growth opportunities giving companies the agility that [2] calls essential to surviving in the volatile modern business environment.

In this paper, we explore the issue of how companies can use immediate employee job assignments to meet current demand and build capacity for the future. We focus on service workers, particularly service technicians. The problem discussed in this paper is a variant of the technician and task scheduling problem (TTSP). In the TTSP, a set of technicians serves a set of customer requests. Customers are associated with certain tasks and different tasks have different skills associated with them. In our version of the problem, technicians have different service times depending on their experience in performing a task 
as well as each technician's ability to transform that experience into improved productivity. We measure experience in the number of times that the technician has performed the task.

The fact that technician productivity, and really all workers productivity, is linked to experience suggests that what could be modeled as a single-period

so problem (i.e. focusing solely on making assignments to serve the current day's tasks) should instead be modeled as a multi-period problem. As such, we consider the multi-period technician scheduling problem that accounts for the fact that productivity increases (or service time decreases) as technicians gain experience. These increases in productivity are often referred to as "learning." We assume that the time that it takes a technician to complete a task depends on the technician's experience in the skill associated with the task and how quickly the technician learns. How quickly a technician learns is known as the technician's learning rate. We assume that we have a set of heterogeneous technicians whose learning rates and initial experience are known. The service time depends 40 on the amount of experience the worker has with the skill required by the task.

We assume that daily demand is not revealed until the day of service. Each day, the technicians serve the day's demand, . In this work, we seek to minimize the expected sum of each day's total service times over a finite horizon. Reflecting what are often tight labor market conditions for technicians (a job 45 definition that can span multiple industries, including home appliance repair, lab technician, and home health care), we choose an objective that seeks to maximize the capacity of an existing workforce. We call our problem variant the multi-period technician scheduling problem with experience-based service times and stochastic customers (MTSP-ESTSC).

To solve the problem, we propose an approximate dynamic programming (ADP) approach that, at each stage, solves a mixed integer program (MIP) to assign technicians to tasks. In addition to recognizing the resulting service times in the current period, the MIP approximates the impact of those assignments on future technician service times (the "cost-to-go") with a forecast of each 55 technician's task assignments in the next period. Assignment decisions in the 
next period are partially driven by each technician's service times on each type of task, which are in turn driven by their experience level on each type of task. To capture this fact, the forecasting model embedded in the MIP is a function of the assignment decisions in the current period.

One of the challenges associated with solving optimization models that recognize that humans learn is that the quantitative models of human learning proposed by the psychology community are non-linear. As a result, to solve a MIP at each stage of the ADP, we adapt an exact reformulation method from the literature that relies on the fact that the function we use to map experience to service time has a finite domain.

In this study, we make the following research contributions. First, we present the first model that explicitly models the impact of individualized, experiencebased learning on the technician scheduling problem. Such a model will facilitate organizational productivity improvement by allowing for more effective workforce management. Further, we discuss how the presented Markov decision process (MDP) model can be adapted to handle cases of worker attrition and new task types. Second, we introduce a method for approximating the future value of today's workforce assignments. With the addition of technician learning to the model, decisions today affect tomorrow's productivity. Thus, 75 the approximation method allows us to do so. Third, using the approximation, we demonstrate how the approximate Bellman equation can be transformed into a linear, mixed integer program. This transformation is significant because the nonlinearity of the learning functions naturally lead to a nonlinear integer programming formulation. Our formulation allows the approximate Bellman equation to be solved by a standard implementation of a commercial integer programming solver.

We demonstrate the value of the proposed solution approach with three experiments. In one experiment, the set of technicians and the set of task types remain the same over the problem horizon. In the second, we introduce a workforce disruption in the middle of the horizon in which one technician leaves the workforce and a new technician is added. The third variant adds an additional 
task type in the middle of the horizon. For each of the three problem variants, we compare the proposed solution approach to a myopic solution approach that views the problem as a single-period problem, ignoring the impact of current period decisions on future service times. Our comparisons demonstrate that the proposed solution approach leads to higher-quality solutions by better positioning technicians to meet future demands.

The remainder of this paper is organized as follows. Section 2 reviews the literature related to the MTSP-ESTSC. Section 3 presents a model for the problem. Section 4 describes the solution approach. Section 5 discuss the design of the experiments, and Section 6 presents our computational results. Finally, Section 7 concludes this work and suggests areas of future research.

\section{Literature Review}

We review the literature of technician scheduling and routing problems as

100

\subsection{Individual Learning and Its Applications}

That humans learn as they gain experience, "learning-by-doing" is a well known phenomenon. The learning effect was first examined on a scientific basis by [3], who quantified learning curves with the observation that in the aircraft

put. Subsequent empirical studies confirmed the existence and importance of learning effects (see for example $[4,5,6,7]$ ). In 2016, the concept has become mainstream enough that it is now included in textbooks on operations management $([8,9])$.

The mathematical descriptions of learning are often called learning curves. Reviews of the literature on learning curves can be found in [10], [11], [12], and [13]. Because of the availability of distributions from which to generate workforces, in this research, we use the hyperbolic learning model described in [14]. We note that, while we employ the hyperbolic learning model, most learn${ }_{115}$ ing curves have similar shapes and would support conclusions similar to those 
discussed in Section 6. We note that there also exists an extensive literature focusing on organizational learning. [15] provides an excellent reference.

Work that explicitly models individual learning and the associated heterogeneity of the workforce demonstrates the value of capturing learning. [16] shows that simply modeling worker heterogeneity without considering learning improves system performance versus assuming uniform workforce productivity in flow-line production. [17] extend the analysis of [16] to demonstrate the impact of heterogeneous learning and forgetting curves on system productivity in a assembly-line setting. [18] confirms the results of [17] for technician routing. In addition to flow lines, assembly lines, and technician routing, the value of modeling learning has also been found in call centers ([19]), departmental assignment ([20]), machine scheduling (see [21] for a review), project selection $([22,23])$, and vehicle routing $([24])$.

One of the challenges of much of the workforce planning literature that models individual learning is that the nonlinearity of the learning curves creates challenges. For this reason, work such as [19] and [25], simplify the model of individual learning to avoid the nonlineariaties. Work such as [26] exploits structural properties of the optimal solution to increase the size of the problem that can be solved. However, such approaches do not generalize. Work such as ${ }_{135}[27],[28]$, and [29] are limited to solving small problems. [30] introduce a linear and integer reformulation of the learning curve that takes advantage of the fact that most work is assigned in time intervals. The reformulation allows much larger problems to be solved than had been previously. We take advantage of the reformulation in this work as well. Other examples of the reformulation can be found in [31], [32], and [33]. Additional review of workforce planning models that incorporate learning can be found in [34] and [30].

\subsection{Technician Scheduling and Routing}

Existing literature contains a variety of technician scheduling and routing problems. A detailed review can be found in [18]. [35] introduce the technician 
into teams. Tasks are assigned to teams so that skill requirements and required skill levels can be matched. In 2007, the French Operations Research Society introduced a challenge (http://challenge.roadef.org/2007/en/) based on [35]'s work and offered a real-world data set for technician scheduling. The challenge resulted in a stream of papers [36, 37, 38, 39]. All of the papers explore deterministic problems and account for neither learning nor the extended horizon over which learning occurs. The multi-period technician scheduling problem with stochastic customers is discussed in [40], but the solution approach is myopic.

A number of papers in the literature consider multi-period scheduling. However, none of these papers incorporates learning effects, and all of the papers study deterministic problems. In contrast, this paper considers that each day's jobs are revealed over time and that the technicians learn from experience. Examples of deterministic, multi-period scheduling problems include $[41,42,43,44,45,46,47,48]$.

Other literature incorporates routing in addition to service time aspects of the problem, but again, does not consider learning and a multi-period horizon. A number of papers treat deterministic variants of the problem $[49,50,51,52$, $53,54]$. A few authors consider stochastic elements of the problem. [55] focuses on uncertainty associated with service times, and [56] on stochastic service travel times. Additional papers incorporate dynamic and stochastic service requests $[57,40,58,59]$. Unlike in this work, however, all theses papers present myopic, reoptimization solution approaches that do not incorporate information about future requests. [60] incorporates information about the future by adapting the sample-scenario approach proposed by [61]. In contrast to the multiple periods studied in this paper, however, [60] focuses serving customers in a one-day period.

We are aware of only one paper in technician routing and scheduling that incorporates learning. [18] consider a multi-period technician routing problem 175 with experience-based learning and stochastic customers. However, unlike in this work, [18] present a myopic solution approach that does not incorporate 
information about future requests in daily decision making.

\section{Problem Formulation}

In this section, we present a formal model for the multi-period technician

Given these parameters, we can determine technician $k$ 's service times for each task in $\mathcal{R}$ at the start of day $t$ using a learning curve. The learning curve requires a technician's experience on a given task up to day $t$. We capture this experience in the $R$-dimensional vector $\mathcal{Q}_{t}^{k}=\left(q_{1 t}^{k}, q_{2 t}^{k}, \ldots, q_{R t}^{k}\right)$, where the $r^{t h}$ entry $q_{r t}^{k}$ indicates technician $k$ 's experience with task $r^{t h}$ at the start of day $t$. We let $\mathcal{Q}_{0}^{k}$ represent technician $k$ 's experience at the beginning of the horizon. Assuming the hyperbolic learning curve [14], technician $k$ 's service time for task 
$r$ on day $t$ is:

$$
d_{r t}^{k}=\left(D_{r}^{k}\left(\frac{q_{r t}^{k}}{q_{r t}^{k}+L_{r}^{k}}\right)\right)^{-1}
$$

Figure 1 is an illustration of the learning curve with $D_{r}^{k}=3.99$ and $L_{r}^{k}=7.65$, showing the negative relationship between technician's experience and service times.

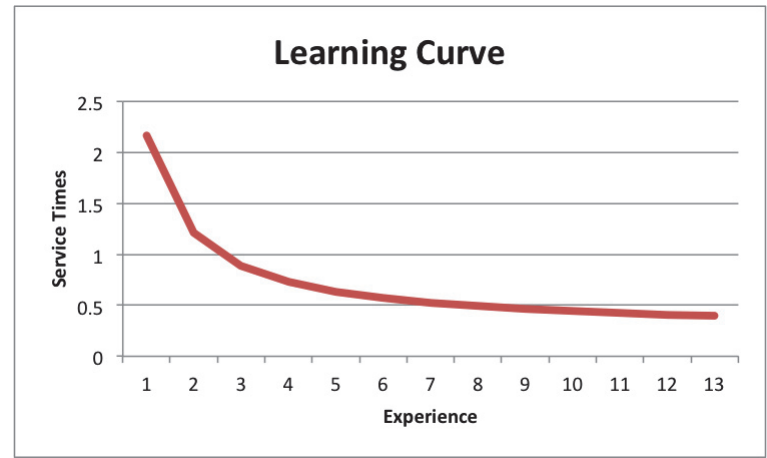

Figure 1: Learning Curve Illustration the experience of the technicians at the start of the day. Then, the state of the system at the beginning of day $t$ is $s_{t}=\left\{\mathcal{N}_{t}, \mathcal{Q}_{t}\right\}$. 
Given state $s_{t}$, an action is a set of assignments that serves the day $t$ requests.

if task $i$ is served by technician $k$ in day $t$. For notational convenience, let $r(i)$ map a task $i$ into the set of task types $\mathcal{R}$. Then, given a day $t$, a feasible action satisfies the following constraints:

$$
\begin{array}{cl}
\sum_{k \in \mathcal{K}} x_{i t}^{k}=1 & \forall i \in \mathcal{N}_{t}, \\
\sum_{i \in \mathcal{N}_{t}} x_{i t}^{k} d_{r(i) t}^{k} \leq C & \forall k \in \mathcal{K} \\
x_{i t}^{k} \in\{0,1\} & \forall i \in \mathcal{N}_{t}, \forall k \in \mathcal{K} .
\end{array}
$$

Constraints (2) ensure that a task is assigned to exactly one technician and that working by the end of the day, and Constraints (4) ensure integrality.

Let $\mathcal{A}_{t}\left(s_{t}\right)$ be the set of all actions available on day $t$ given state $s_{t}$. We assume that each technician's learning is deterministic and based on accumulated experience. Therefore, given that the state is currently $s_{t}$ and that action $a_{t}$ $s_{t}^{a}=\left\{\mathcal{N}_{t}, \mathcal{Q}_{t}^{a}\right\}$ by updating technicians' experience as follows:

$$
q_{r, t+1}^{k}\left(s_{t}, a_{t}\right)=q_{r t}^{k}+\sum_{i \in \mathcal{N}_{t}: r(i)=r} x_{i t}^{k} \quad \forall k \in \mathcal{K}, \forall r \in \mathcal{R}
$$

Recalling that experience is measured as the number of times that the technician has performed the task. Equation (5) adds each technician's existing experience the experience gained from the day $t$ assignments.

We let $W_{t+1}=\left(w_{1, t+1}, \ldots, w_{\check{N}_{t+1}, t+1}\right)$ be the random exogenous information for day $t+1$, where $w_{i, t+1}$ is a random variable representing the task type of the $i^{t h}$ service request on day $t+1$ and $\check{N}_{t+1}$ is a random variable representing the random number of requests on day $t+1$, neither of which is known prior to day $t+1$. Following day $t$, we observe the exogeneous information for day $t+1$ 
from post-decision state $s_{t}^{a}$ to pre-decision state $s_{t+1}=\left\{\mathcal{N}_{t+1}, \mathcal{Q}_{t+1}\right\}$, where $\mathcal{N}_{t+1}$ is the realization of the exogenous process for day $t+1$. In this transition, technicians' experience remain unchanged, thus $\mathcal{Q}_{t+1}=\mathcal{Q}_{t}^{a}$. The technicians' productivity for day $t+1$ can be calculated according to the learning curve given the technicians' experience levels.

At decision epoch $t$, given state $s_{t}$ and action $a_{t}\left(s_{t}\right)$, a transition from predecision state $s_{k}$ to post-decision state $s_{k}^{a}$ results in a contribution

$$
C\left(s_{t}, a_{t}\right)=\sum_{k \in \mathcal{K}} \sum_{i \in \mathcal{N}_{t}} x_{i t}^{k} d_{r(i) t}^{k} \quad \forall a_{t} \in \mathcal{A}_{t}\left(s_{t}\right),
$$

which is the total service time to complete all tasks on day $t$. The problem objective is then $\min _{\pi \in \Pi} E\left[\sum_{t=1}^{T} C\left(s_{t}, \delta_{t}^{\pi}\left(s_{t}\right)\right)\right]$, where $\pi$ is a policy that determines actions for all days $t$ over the problem horizon $T, \Pi$ is the set of all policies, and $\delta_{t}^{\pi}\left(s_{t}\right)$ is a decision rule specific to policy $\pi$ that maps the state $s_{t}$ to an action ${ }_{245} a_{t}$.

In our experiments, we consider two problem variants: (1) attrition and hiring in the technician pool and (2) a new task type to be serviced. We can accommodate this variants with minor changes to the model. In the attrition and hiring scenario, we assume that a technician $k$ 's decision to leave the workforce is exogenous to our model. As such, we model such a departure as an addition to the exogenous information that is observed the day after a technician's departure. Thus, when technician $k$ leaves the workforce just after day $t-1$ and is replaced by a new technician $k^{\prime}$ on day $t$, we update $\mathcal{Q}_{t}$ by setting $q_{r, t}^{k}\left(s_{t}, a_{t}\right)=I_{r}^{k^{\prime}}$, where $I_{r}^{k^{\prime}}$ is the initial experience level of technician $k^{\prime}$ on 255 tasks of type $r$ for each task type $r$.

We handle the second variant similarly. Specifically, for the second variant wherein a new task type (labeled $R+1$ ) is to be serviced starting on day $t$, in the transition from day $t-1$ to day $t$, we expand the state to include the experience levels $q_{R+1, t}^{k}$, for all $k$ in $\mathcal{K}$. Then, the value of $q_{R+1, t}^{k}$ is initialized to whatever the appropriate value should be for each technician. 


\section{Solution Approach}

Given our assumptions, an optimal policy can be found by using the wellknown Bellman equation. The general form of the Bellman equation and the form sufficient for our problem is given by setting

$$
V\left(s_{t}\right)=\min _{a \in \mathcal{A}_{t}\left(s_{t}\right)}\left\{C\left(s_{t}, a\right)+E\left[V\left(s_{t+1}\right) \mid s_{t}, a\right]\right\} .
$$

While we can easily state the Bellman equation for our problem, because of the need to capture each day's set of requests in the state space, the problem is far too large for an exact solution approach such as backward dynamic programming. Instead, we introduce an approximate dynamic programming (ADP) approach that approximates the cost-to-go, the second term in Equation (7), using a forecast of technician assignments for one day in the future. Using this approximation, we then step forward in time. Analogous to rollout algorithms (see [63]), by stepping forward in time, we need only find actions for states that are realized. In the remainder of this section, we first present our approximation of the cost-to-go. We then demonstrate how to use this approximation to solve an approximate form of the Bellman Equation, Equation (7). Finally, we discuss our forward ADP algorithm.

\subsection{Cost-to-Go Approximation and Solving the Approximate Bellman Equation}

For each technician, this forecast is based on the technician's previous assignments and a forecast of the next day's tasks. Specifically, for each technician and each task type, we first predict the demand requests for the next period with an exponential smoothing forecasting: $F_{r t}^{k}=\alpha F_{r, t-1}^{k}+(1-\alpha) A_{r, t-1}^{k}$, where $F_{r, t-1}^{k}$ and $A_{r, t-1}^{k}$ are the numbers of predicted and actual type $r$ demand re280 quests assigned to technician $k$ for period $t-1$, respectively, and $F_{r t}^{k}$ is the forecasted number of assignments of task type $r$ to technician $k$ on day $t$. The parameter $\alpha$ is the smoothing factor. Given an assignment of technician $k$ for each task $i$ on day $t-1, x_{i, t-1}^{k}$, we can compute $A_{r, t-1}^{k}$ as $\sum_{i \in \mathcal{N}_{t-1}: r(i)=r} x_{i, t-1}^{k}$. 
In addition, given an assignment of technician $k$ for each task $i$ on day $t-1$, $x_{i, t-1}^{k}$, we can compute $\hat{d}_{r t}^{k}$, the forecasted service time on day $t$ for task type $r$ and technician $k$. For technician $k$ and task type $r, \hat{d}_{r t}^{k}$ can be calculated using the assignments for day $t-1$ as:

$$
\hat{d}_{r t}^{k}=\left(D_{r}^{k}\left(\frac{q_{r, t-1}^{k}+\sum_{i \in \mathcal{N}_{t-1}: r(i)=r} x_{i, t-1}^{k}}{q_{r, t-1}^{k}+\sum_{i \in \mathcal{N}_{t-1}: r(i)=r} x_{i, t-1}^{k}+L_{r}^{k}}\right)\right)^{-1} .
$$

With the forecasted number of assignments for day $t, F_{r t}^{k}$ and forecasted service times, for each technician $k$ and task type $r$, we can write an approximate Bellman equation as:

$$
\hat{V}\left(s_{t-1}\right)=\min _{a \in \mathcal{A}_{t}\left(s_{t-1}\right)}\left\{C\left(s_{t-1}, a\right)+\sum_{k \in \mathcal{K}} \sum_{r \in \mathcal{R}} F_{r t}^{k} \hat{d}_{r t}^{k}\right\}
$$

Equation (9) can be rewritten as a math program as follows:

$$
\text { (F) } \min \sum_{k \in \mathcal{K}} \sum_{i \in \mathcal{N}_{t-1}} x_{i, t-1}^{k} d_{r(i), t-1}^{k}+\sum_{k \in \mathcal{K}} \sum_{r \in \mathcal{R}} F_{r t}^{k} \hat{d}_{r t}^{k}
$$

subject to $(2),(3),(4)$

$$
F_{r t}^{k}=\alpha F_{r, t-1}^{k}+(1-\alpha) \sum_{i \in \mathcal{N}_{t}: r(i)=r} x_{i, t-1}^{k} \quad, \forall k \in \mathcal{K}, \forall r \in \mathcal{R},
$$

$$
\hat{d}_{r t}^{k}=\left(D_{r}^{k}\left(\frac{q_{r, t-1}^{k}+\sum_{i \in \mathcal{N}_{t-1}: r(i)=r} x_{i, t-1}^{k}}{q_{r, t-1}^{k}+\sum_{i \in \mathcal{N}_{t-1}: r(i)=r} x_{i, t-1}^{k}+L_{r}^{k}}\right)\right)^{-1}, \forall r \in \mathcal{R}, \forall k \in \mathcal{K},
$$

$$
x_{i, t-1}^{k} \text { binary } \quad, \forall k \in \mathcal{K}, \forall i \in \mathcal{N}_{t-1} .
$$

The objective is to minimize the sum of total service time and the forecasted service time for the next period. As presented earlier, Constraints (2) to (4) define feasible actions. Constraints (10) are replaced with $F_{r t}^{k}=\sum_{i \in \mathcal{N}_{t}: r(i)=r} x_{i, t-1}^{k}$ when $t-1=1$. Constraints (11) determine the service time for the forecasted 
assignments.

Model $(\mathbf{F})$ is non-linear. We use the reformulation presented by [30] to overcome this challenge. The reformulation takes advantage of the fact that the task assignments and thus experience are discrete and that the maximum number of tasks of each type is known for a given day $t-1$. Thus, we can enumerate the set of potential forecasted service times for each technician on each task type for day $t$. We describe the steps of the enumeration in Algorithm 1.

To present the reformulation, we first present some additional notation. We let $z_{r, t-1}^{k j}$ be a binary variable indicating whether technician $k$ did $j$ tasks of type $r$ on day $t-1$. Let $\bar{d}_{r t}^{k j}$ represent technician $k$ 's estimated service times for serving tasks of type $r$ on day $t$ having done $j$ jobs of task $r$ on day $t-1$. Then, having completed $j$ tasks of type $r$ on day $t-1$ and given $\bar{d}_{r t}^{k j}$ for technician $k$ on task type $r$ for day $t$, the total time required for $k$ to complete the forecasted number of tasks of type $r$ on day $t$ is $\overline{S T}_{r t}^{k j}=\bar{d}_{r t}^{k j} F_{r t}^{k}$. We introduce $S T_{r t}^{k}$ as a decision variable that gives the total service time of technician $k$ on tasks of type $r$ for day $t$. Finally, let $M_{t-1}=\left(m_{1, t-1}, m_{2, t-1}, \ldots, m_{R, t-1}\right)$ be a vector of the number of each task type $r$ for day $t-1$. Then, the reformulation model is as follows:

$$
\text { (R) } \min \sum_{k \in \mathcal{K}} \sum_{i \in \mathcal{N}_{t-1}} x_{i, t-1}^{k} d_{r(i), t-1}^{k}+\sum_{k \in \mathcal{K}} \sum_{r \in \mathcal{R}} S T_{r t}^{k}
$$

subject to $(2),(3),(4),(10)$

$$
\begin{array}{ll}
S T_{r t}^{k}=\sum_{j=1}^{m_{r, t-1}} \overline{S T}_{r t}^{k j} z_{r, t-1}^{k j} & , \forall r \in \mathcal{R}, \forall k \in \mathcal{K} \\
\sum_{j=1}^{m_{r, t-1}} j z_{r, t-1}^{k j}=\sum_{i \in \mathcal{N}_{t-1}: r(i)=r} x_{i, t-1}^{k} & , \forall r \in \mathcal{R}, \forall k \in \mathcal{K} \\
\sum_{j=1}^{m_{r, t-1}} z_{r, t-1}^{k j}=1 & , \forall r \in \mathcal{R}, \forall k \in \mathcal{K} \\
z_{r t}^{k j} \text { binary } & , \forall r \in \mathcal{R}, \forall k \in \mathcal{K}, j=1, \ldots, m_{r t} .
\end{array}
$$




\subsection{Forward Approximate Dynamic Programming Algorithm}

Algorithm 1 presents our forward ADP algorithm for solving instances of the MTSP-ESTSC. Unlike the traditional backward dynamic programming algorithm, Algorithm 1 steps forward in time. Stepping forward in means offers that are actually visited. However, stepping forward in time also means that we have not computed values for the cost-to-go in the Bellman equation. We instead approximate the cost-to-go in the manner described in the previous section. Using that approximation and the previously described reformulation, the approximate Bellman equation given in Equation (9) can be solved using the math program given in $\mathbf{R}$.

Algorithm 1 outputs a series of actions, or technician assignments, one for each state visited over the planning horizon. The algorithm takes as input the set of technicians, their learning parameters, and the set of task types. Line (3) initializes the state by setting each technicians experience on each task type to that technician's given initial experience.

Then, the algorithm seeks to determine the set of assignments for each technician for each day. At the beginning of each day, the day's service requests are observed (Line (5)). Then, Lines (6) to (13) enumerate the possible total 320 forecasted service times for day $t+1$. With these enumerated values, Line (14) solves the resulting instance of math program $\mathbf{R}$. 


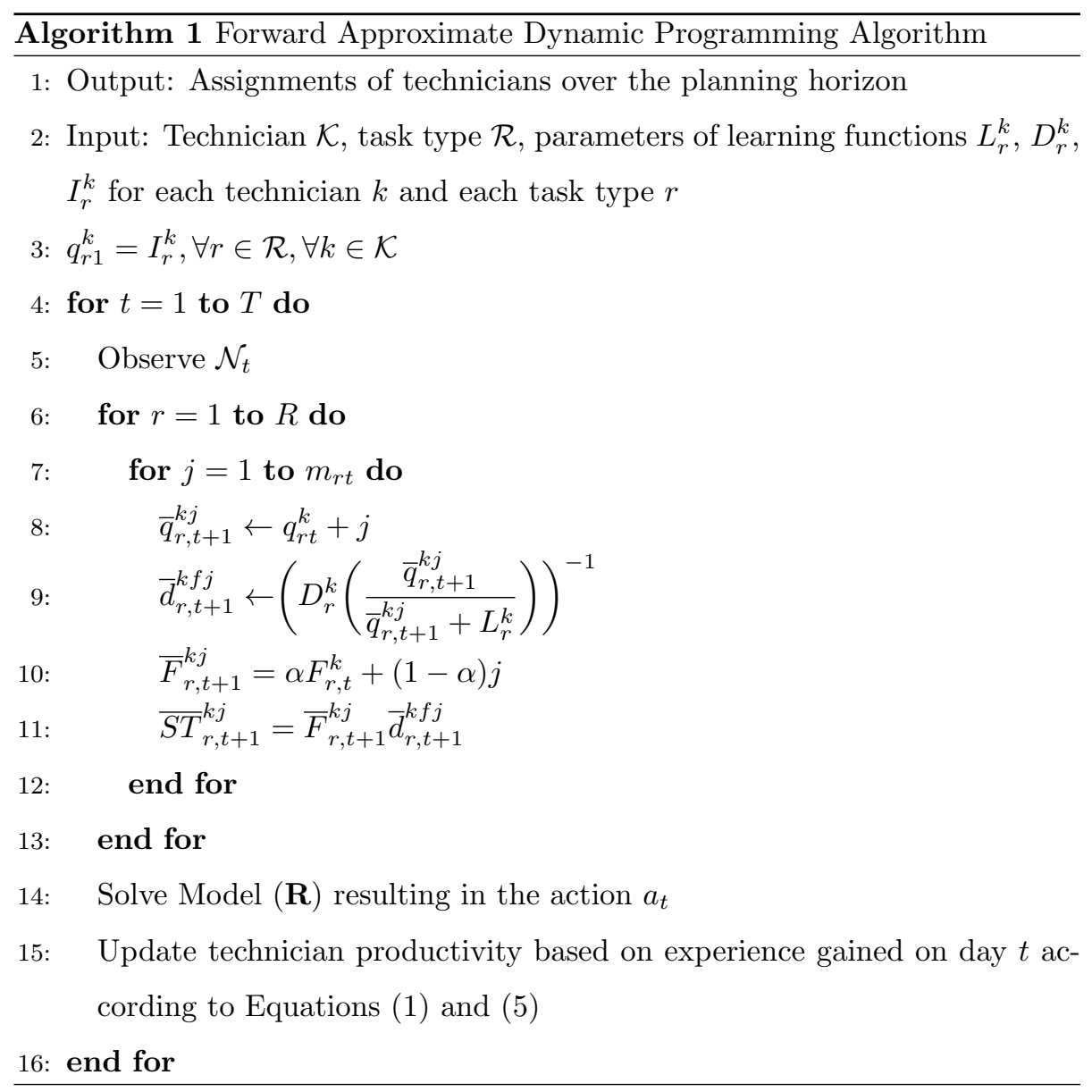

\section{Experimental design}

In this section, we describe the instances that we create to computationally test the value of our solution approach. Our instances all involve one of 30 325 workforces of five technicians with heterogeneous learning parameters. In particular, the learning rate and steady state productivity rate are sampled from a multivariate normal distribution with the mean and covariance matrix derived from empirical data by [14], given in Table 1 . 


$$
\begin{gathered}
\mu^{T}=\left(\begin{array}{llll}
\log D & \log I & \log L & F \\
1.448 & 1.963 & 2.0446 & 2.269
\end{array}\right) \\
\Sigma= \\
\quad \begin{array}{ccccc}
\log D & \log I & \log L & F \\
\log D \\
\log I \\
\log L \\
F
\end{array}\left(\begin{array}{ccccc}
0.0045 & 0.0167 & 0.0191 & -0.0214 \\
0.0167 & 0.4621 & 0.2443 & 0.2380 \\
0.0191 & 0.2443 & 0.1905 & 0.2326 \\
-0.0214 & -0.2380 & -0.2326 & 0.3638
\end{array}\right)
\end{gathered}
$$

Table 1: Mean and covariance of workforce parameters

For each workforce, we consider four levels of capacity $(C)$. These levels are levels represent the maximum amount of time that a technician can work in a day. We also assume four different cardinalities for the set of task types $\mathcal{R}$. We refer to these levels as task diversity. We consider four levels of task diversity $(R)$ : 5, 10, 15, and 20. With 30 workforces, four levels of workforce capacity, 335 and four levels of task diversity we have 480 instances in total.

We test the 480 instances on 120-day horizons. To limit the number of factors in our experimental design, we assume 50 task requests per day. For each of the 480 instances, we generate 100 trials of the 120-day horizon. To generate a trial, for each day, each of the 50 requests is randomly assigned a task type using a discrete uniform distribution.

We also conduct experiments regarding the two problem variants discussed in Section 3. For the first, we model a disruption in the workforce wherein one technician leaves the workforce and a new technician is hired as a replacement in the middle of the planning horizon. Specifically, Technician 1 is replaced with a new technician with initial experience level on all types of tasks on day 61. Specifically, on day 61 , the transition function for Technician 1 is $q_{r, 61}^{1}\left(s_{60}, a_{60}\right)=I_{r}^{1}$ for every $r$ in $\mathcal{R}$.

For the second variant, we consider the situation in which a new task type is 
introduced in the middle of the planning horizon. We assume that all technicians are inexperienced on this new task type, and we set their experience levels on the new task type to be one on the day of the introduction (day 61 ). Specifically, on day 61 , the transition function for the new task type is $q_{R+1,61}^{k}\left(s_{60}, a_{60}\right)=$ $1 \forall k \in \mathcal{K}$. In both the case of workforce disruption and the new task type, we use capacity levels of $9,10,11$, and 12 to ensure the feasibility.

With these instances, we consider three separate experiments. In all three experiments, we seek to demonstrate the value of our method for approximating the future value of current decisions. In the first set of experiments, we consider the 480 instances described above and solve each with the proposed ADP approach. As a benchmark, we present solutions from a myopic approach that approximates the Bellman equation by setting the second term of Equation (7) to 0 . The resulting daily optimization problem can then be solved by solving a simplified version of the math program $\mathbf{R}$. Our choice of benchmark is motivated by the work in [18] that demonstrated that the difference between the quality of solutions from models that incorporate learning and those that do not are significant. The second and third experiments are analogous to those just described except that we solve the workforce disruption and new product variants, respectively.

All computations are performed by Gurobi 5.6 with Python 2.7 interface on Intel Xeon processor running at CentOS 6.3. In addition, for all experiments, we 370 set the smoothing factor $(\alpha)$ to $\alpha=0.9$. This value was chosen after examining the results of various choices of $\alpha$ over many different instances.

\section{Computational Analysis}

In this section, we present the results of our computational experiments. We do not report runtimes as the math program $\mathbf{R}$ solves nearly instantaneously 375 and is thus capable of providing daily technician assignments in a timely fashion. 


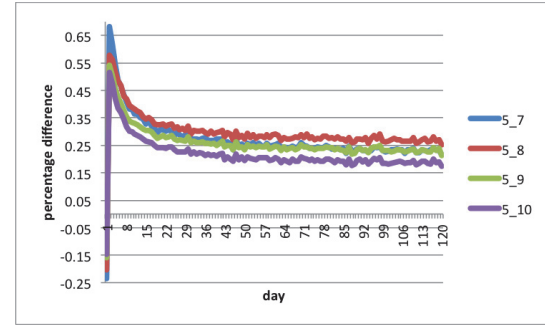

(a) Diversity $=5$

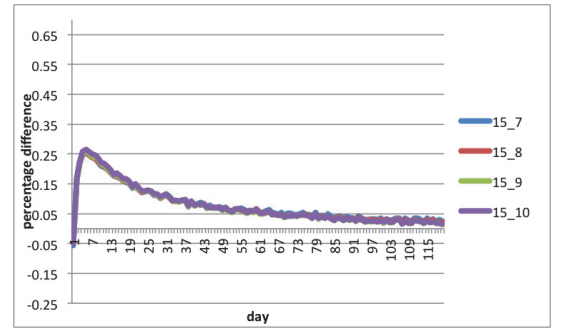

(c) Diversity $=15$

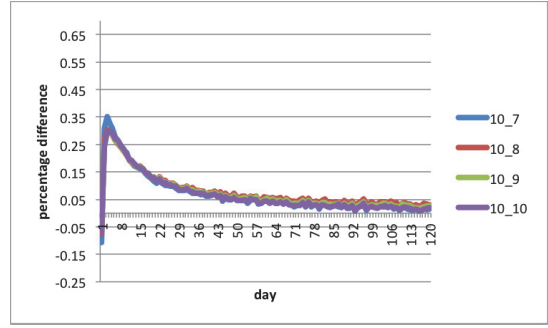

(b) Diversity $=10$

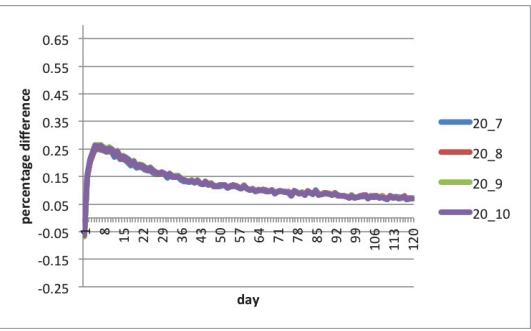

(d) Diversity $=20$

Figure 2: Average daily gaps by task diversity (\%)

\subsection{The Value of Incorporating Information about Future Requests}

In this first experiment, we solve the 120-day MTSP-ESTSC with the ADP algorithm and the previously described myopic approach. Figures 2 and 3 present the results of the experiment. The figures present the average daily differences between the two solution approaches, averaged over all capacity and diversity levels, respectively. Specifically, for both ADP and myopic approaches, we record the total service time for each day of the 120-day horizon for each trial (using common trials between the two approaches). We record these values as $O b j_{m}^{t}$ and $O b j_{p}^{t}$ for the myopic and ADP approaches, respectively. We then compute the objective gap on day $t$ as $g a p_{m-p}^{t}=\frac{o b j_{m}^{t}-o b j_{p}^{t}}{o b j_{p}^{t}} \times 100 \%$ for each trial. For Figure 2, for each diversity level, the results are presented for each diversity level averaged over all capacity levels. Analogously, for Figure 3, the results are presented for each capacity level averaged over all diversity levels.

For all task diversities and all capacity levels, the average daily gap follows a similar trend. From Figures 2 and 3, we see a sharp increase from negative to positive gaps for the first three to eight days depending on the diversity levels. 


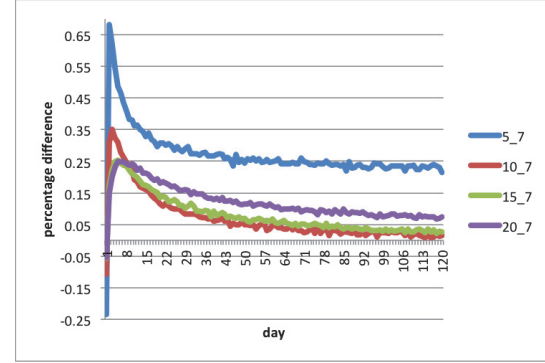

(a) Capacity $=7$

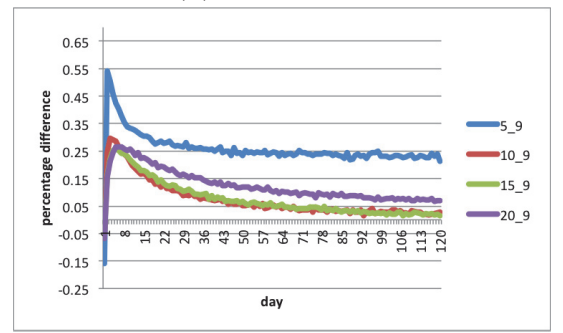

(c) Capacity $=9$

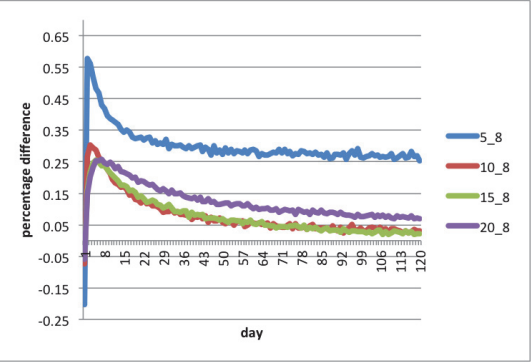

(b) Capacity $=8$

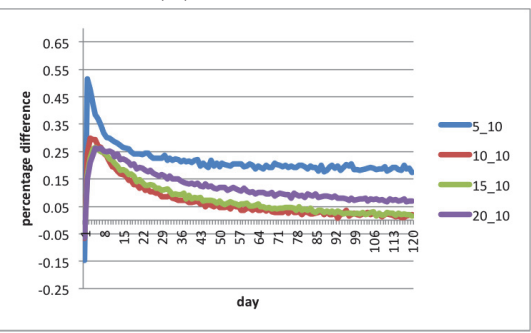

(d) Capacity $=10$

Figure 3: Average daily gaps by capacity (\%)

The myopic approach focuses on optimizing only current period service times while the ADP algorithm balances current and next periods so as to achieve the best assignment over both current and next periods. Then, the objective value on day one is not optimal with the ADP algorithm. In addition, there is no history information on day one, so the ADP approach has no advantage over the myopic approach. The above two reasons explain the negative gap on day one. As it accumulates more information over time, the ADP approach shows an increasing advantage, which is reflected by the sharp increase of the positive gaps between the two approaches. According to learning curves, most learning effects occur during the early periods, which corresponds to the larger gaps between the myopic and ADP earlier in the horizon. Then, as the technicians accumulate more experience and approach the plateau of the learning curves, the average daily gaps decrease slowly to a relatively stable level.

Comparing the performance of the ADP algorithm and the myopic approach across task diversity levels (Figure 2), we see that the benefits associated with 
looking into the future when performing task assignments are negatively correlated with diversity level; the lower the diversity level, the greater the benefit. We hypothesize that there are two reasons for this behavior: (1) with a lower task diversity level our forecasting model is more accurate as there is more historical data for each task type, and, (2) with a lower task diversity level, as there are many tasks of each type, we are able to fully leverage the benefits of learning more quickly by looking into the future.

Comparing the performance of the ADP algorithm and the myopic approach across capacities (Figure 3), we see that the benefits associated with looking into the future when performing task assignments are negatively correlated with capacity; the lower the capacity level, the greater the benefit. An examination of the results suggests that the myopic approach seeks to assign each task to the technician that can perform it the quickest. However, the ability of the approach to do so is especially limited when capacity is tight. We hypothesize that, by looking into the future, the ADP algorithm recognizes the value of developing the proficiency level of multiple technicians so that many of them are able to perform each task quickly. As a result, as it positions the workforce so that it can assign tasks to different technicians that have the same proficiency, the 425 ADP algorithm outperforms the myopic approach the most at lower capacity levels.

We also study the distribution of the average daily gap among instances. Table 2 shows the number of instances with positive, negative and zero average daily gap for all diversities and capacities, respectively. Results indicate that there are more instances with positive gap than those with negative gap and two approaches are more likely to perform the same in terms of the gap with loose capacity. This result is not surprising as, when technicians are allowed to serve more tasks per day, the faster learners and more experienced technicians are assigned to more tasks. 


\begin{tabular}{lllllllllllll}
\multicolumn{4}{c}{ Positive gap } & \multicolumn{4}{c}{ Negative gap } & \multicolumn{4}{c}{ Zero gap } \\
\hline & $\mathrm{R}=5$ & $\mathrm{R}=10$ & $\mathrm{R}=15$ & $\mathrm{R}=20$ & $\mathrm{R}=5$ & $\mathrm{R}=10$ & $\mathrm{R}=15$ & $\mathrm{R}=20$ & $\mathrm{R}=5$ & $\mathrm{R}=10$ & $\mathrm{R}=15$ & $\mathrm{R}=20$ \\
$\mathrm{C}=7$ & 1074 & 1055 & 926 & 1237 & 455 & 518 & 599 & 365 & 1471 & 1427 & 1475 & 1398 \\
$\mathrm{C}=8$ & 1104 & 907 & 793 & 1112 & 306 & 517 & 586 & 385 & 1590 & 1576 & 1621 & 1503 \\
$\mathrm{C}=9$ & 977 & 836 & 761 & 1065 & 348 & 511 & 572 & 352 & 1675 & 1653 & 1667 & 1583 \\
$\mathrm{C}=10$ & 773 & 792 & 760 & 1064 & 401 & 501 & 573 & 361 & 1826 & 1707 & 1703 & 1575 \\
\hline
\end{tabular}

Table 2: Distribution of the average daily gaps

\subsection{Responding to a Disruption in the Workforce}

In this subsection, we present the results of the experiments designed to identify the impact of a disruption in the workforce. Figure 4 illustrates the changes of average daily gap by diversity level. The gaps are computed analogously to those presented in Figures 2 and 3. The general trends of the average daily gap changes are similar to those in the previous experiments, except for the temporary fluctuations on and after the day in which one technician departs and a new technician joins the workforce (day 61-70). The performance of the ADP approach after the disruption mimics its performance at the beginning of the planning horizon, where we see a transition from a negative to a positive longer service times in the near-term. 


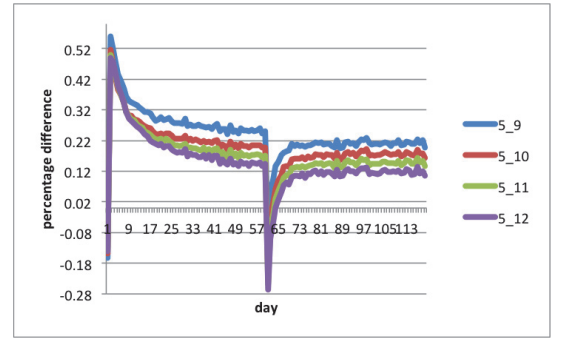

(a) Diversity $=5$

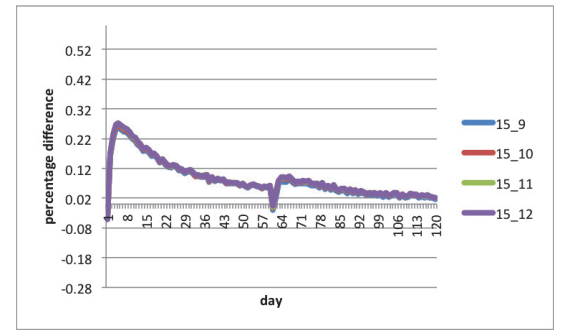

(c) Diversity $=15$

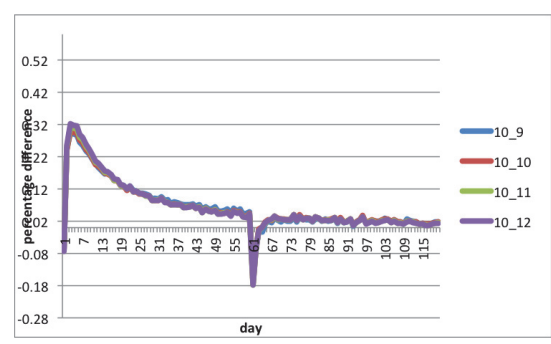

(b) Diversity $=10$

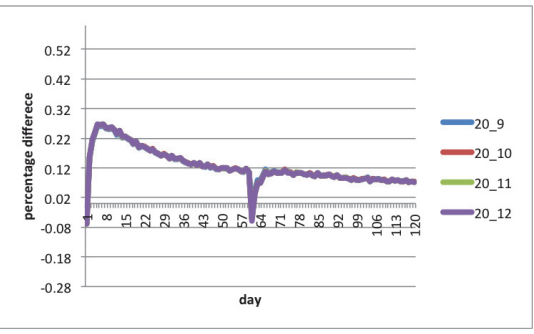

(d) Diversity $=20$

Figure 4: Average daily gaps by task diversity (\%) with workforce fluctuation

Next, we study the new technician's workload (i.e. Technician 1 in our experiment). Figures $5 \mathrm{a}$ and $5 \mathrm{~b}$ present the average workload of the new technician (as a percentage of the 50 tasks served) over the horizon (days 61-120) and on the day of disruption (day 61). We see that in both cases the ADP algorithm assigns more work to the new technician than the myopic approach does. Just as at the beginning of the planning horizon, the ADP algorithm is positioning the new technician to have sufficient experience for task requests on future days, something the myopic approach ignores. In fact, in some cases the new technician does more than the average $(20 \%)$ of the work. We hypothesize that in these cases the ADP approach is aggressively building up the experience level of the new technician. 


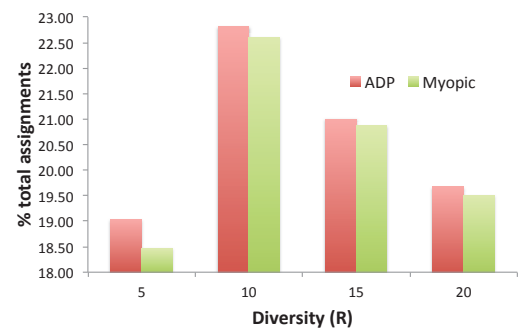

(a) Over horizon

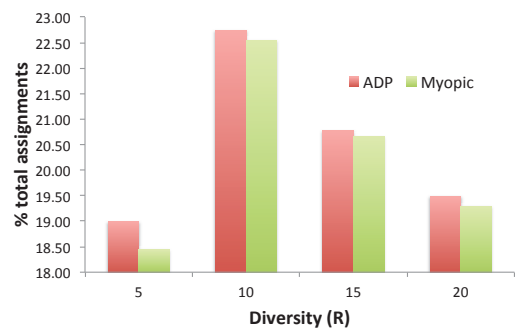

(b) On day of disruption

Figure 5: Workload of new technician

We also note that the lower the task diversity the larger the negative gaps when introducing the new technician (see the deep plunge on day 61). With a lower task diversity, there are more tasks for each task type, which in turn leads to technicians rapidly accumulating enough experience to operate at their maximum productivity. As such, there is a greater difference in the service times between the new technician and the existing workforce on the day the new technician begins. Yet, as discussed above, the ADP approach assigns more work to the new technician than the myopic approach to better prepare the new technician for future task requests. This impact of diversity is most apparent at the lowest level $(\mathrm{R}=5)$. Here, we see the greatest difference in the workload assigned to the new technician as the myopic approach is focused solely on leveraging the productivity of the existing, highly-experienced technicians.

\subsection{Responding to a New Task Type}

We next present results for the experiments that examine the impact of introducing a new task. Figure 6 illustrates average daily gaps by day and diversity. The gaps are computed analogously to those in Section 6.1. We notice that the general trends of the average daily gap remain the same as those seen in Section 6.2. As with attrition, after a new task type is introduced the ADP algorithm accepts longer service times to better position the workforce to handle task requests for the new task type in the future. 


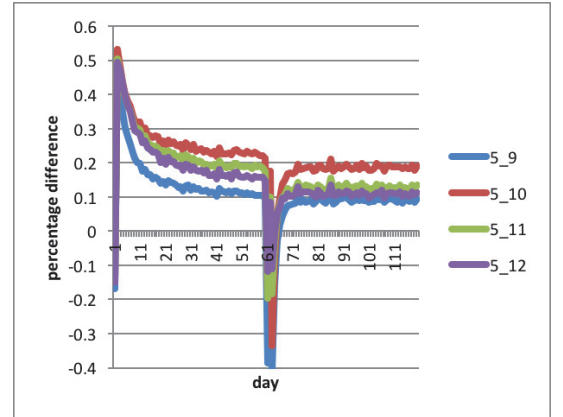

(a) Diversity $=5$

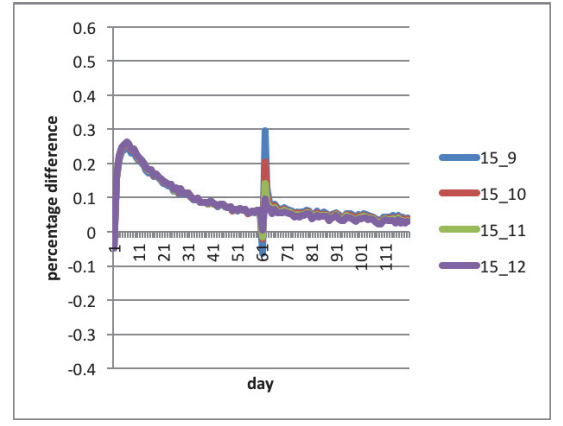

(c) Diversity $=15$

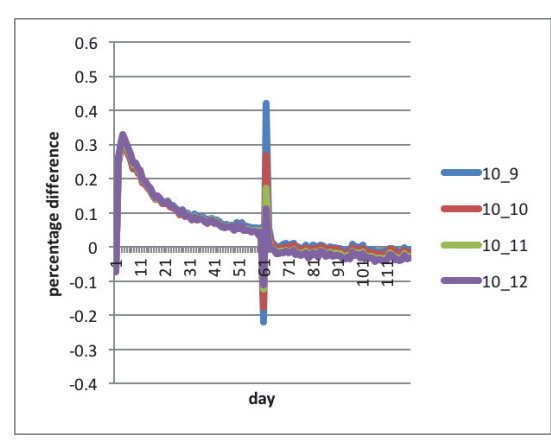

(b) Diversity $=10$

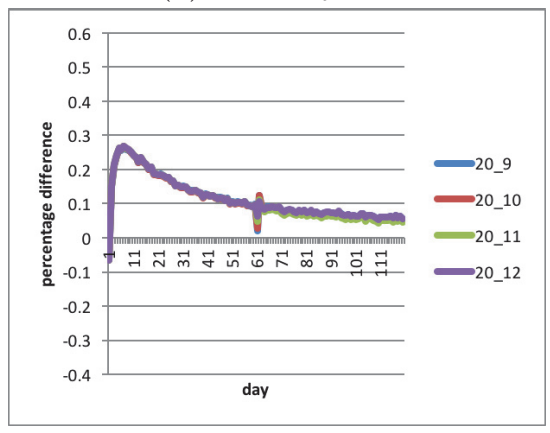

(d) Diversity $=20$

Figure 6: Average daily gaps by task diversity (\%) with a new task type

Next, for each approach (the myopic and the ADP), we examine the distribution of experience in the new task type. Specifically, for each workforce and each instance, we calculate the percentage of times that the tasks of the new type are performed by the technician who performs them the most often (who we will call the most frequent technician). Similarly, we calculate the percentage of times that the tasks of the new type are performed by the technician who performs them the second most often (and third most, fourth most, fifth most). We report in Figure 7 the percentage of times for each approach and diversity level tasks of the new type are performed by the most frequent technician. As these new type tasks are very rarely (e.g. $<.65 \%$ of the time) performed by the technician who performs it the third (or fourth or fifth) most often, one can reasonably conclude that, when the most frequent technician does not perform the task type, it is performed by the second most frequent technician. 


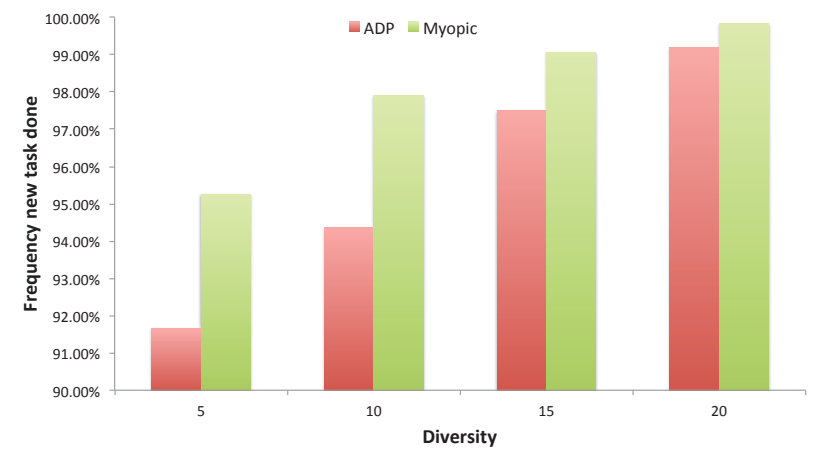

Figure 7: How often most frequent technician performs tasks of new type

What we observe in Figure 7 is that both approaches favor having one individual specialized on tasks of the new type and that specialization is directly correlated to the diversity of the instance (i.e. the number of different task types). However, the ADP approach also builds proficiency in the new task type in a second technician and does so more often when diversity is low. Similar to the discussion above, we hypothesize that this is because, at lower diversity levels, technicians can quickly develop proficiency, due to the number of opportunities, in the original task types. Thus, it is less impactful to divert two of them towards building proficiency in the new task type.

\section{Conclusions and Future Work}

In this paper, we study the scheduling problem of assigning tasks to technicians wherein the tasks to perform each day are not known until the day they are to be done. Such a problem can be modeled as a daily, task assignment problem. However, we also model that technicians learn. That is, technicians' service times decrease as technicians gain experience performing tasks. As such, we model the problem as a multi-period problem in which each day's decisions are made with an eye to building capacity (via experience accumulation) for the future. This problem, which we call the multi-period technician scheduling problem with experience-based service times and stochastic tasks, is new to the 
cuss how it can be adapted to disruptions in the workforce and the introduction of new task types to service.

We present an approximate dynamic programming-based solution approach for this model that repeatedly solves optimization problems to make daily as-

literature. We present a Markov decision process model for the problem and dissignments that recognize the impact of technicians gaining experience on future service times. The optimization problem approximates the cost-to-go with forecasts of the next day's assignments for each technician and the resulting estimated time it will take to service those assignments given the current day's decisions. As quantitative models of human learning are non-linear, we use a technique from the literature to reformulate these optimization problems to mixed integer programs which can then be solved (nearly) instantaneously.

We demonstrate the value of both the model and the solution approach with an extensive computational study. We benchmark the performance of the model and ADP-based solution approach against a myopic approach that models the problem as a single-period problem, ignoring the impact of experience gained today on service times on later days. We see that across all variants (the baseline problem, disruption in workforce, and introduction of new task types) the ADPbased approach outperforms the myopic approach by sacrificing solution quality in a few near-term periods to better position the workforce for future task service requests.

There are a number of avenues for future work. First, as noted in Section 3, there are a number of factors other than experience that can affect an individual's ability to perform a task. Such considerations include learning from similar task [62], learning by transfer from other technicians [64], the training received $53565,66]$, and even the technician's workload $[67,68]$. While industrial psychologists have not completely identified how to measure all of these effects, including elements such as these in the formulation could offer additional insights into the problem. Second, the currently proposed cost-to-go approximation estimates the value of experience accumulation through today's assignments only one day into the future. Future work could explore alternate methods that approximate 
that value farther into the future. Also, many technician-related scheduling problems include a routing component. It would be interesting to test the value of the proposed (or a similar) approach on such a problem. Finally, an analysis of the structure of solutions produced by an ADP-based solution approach could yield interesting insights into how schedulers should make these assignment decisions on a daily basis.

\section{Appendix}

We have examined the results of various choices of $\alpha$ over many different instances with our base experiment. We test $\alpha$ values of $0.1,0.3,0.5,0.7$, and 0.9 .

Figures 8 and 11 present the average daily differences between the approximate dynamic programming and myopic approaches with different values of $\alpha$.

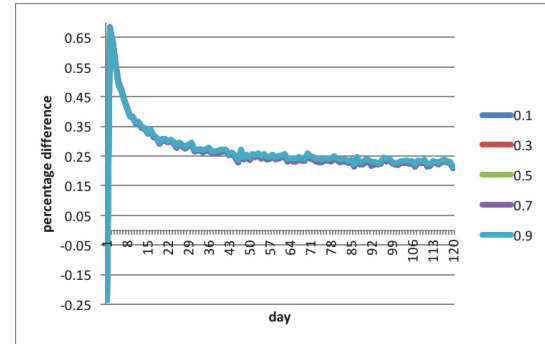

(a) Capacity $=7$

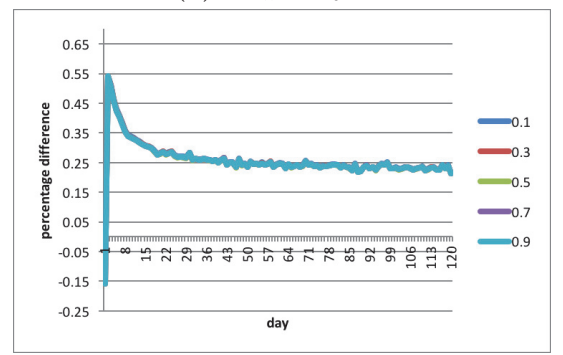

(c) Capacity $=9$

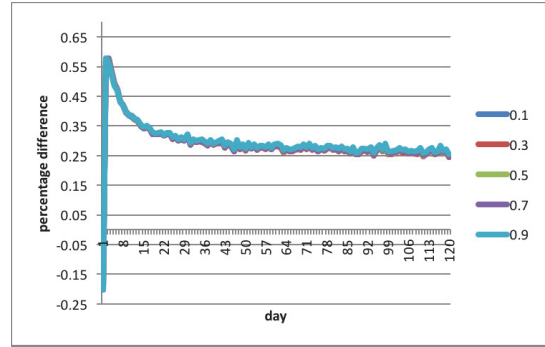

(b) Capacity $=8$

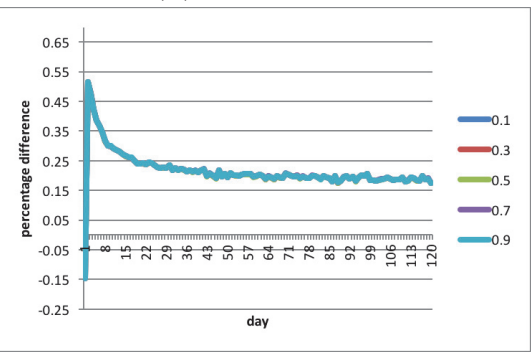

(d) Capacity $=10$

Figure 8: Average daily gaps (\%) for diversity $=5$ 


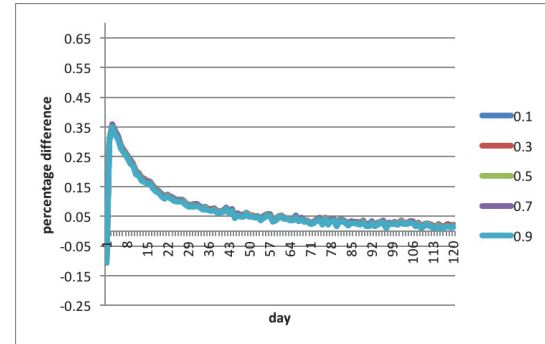

(a) Capacity $=7$

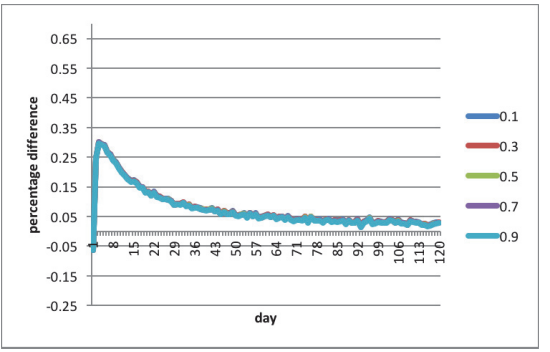

(c) Capacity $=9$

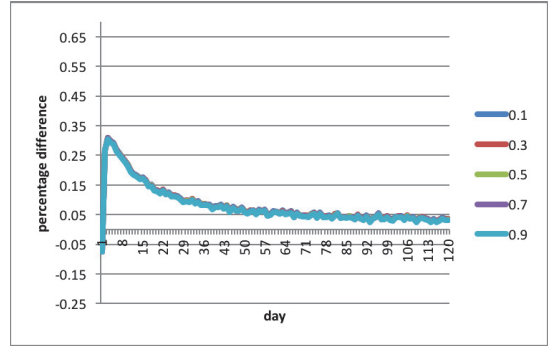

(b) Capacity $=8$

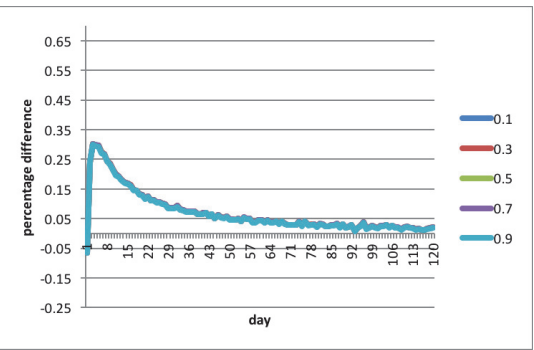

(d) Capacity $=10$

Figure 9: Average daily gaps (\%) for diversity=10

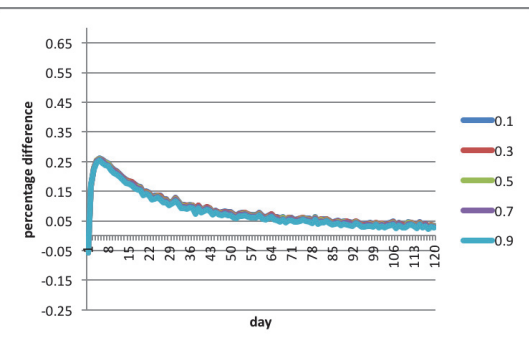

(a) Capacity $=7$

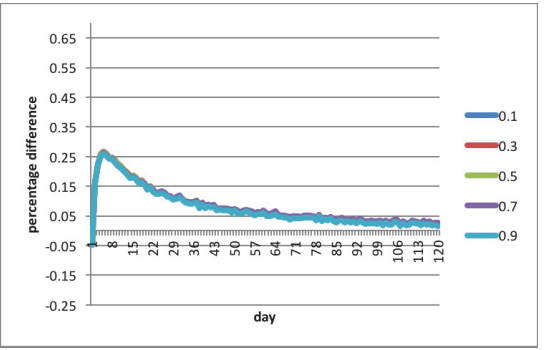

(c) Capacity $=9$

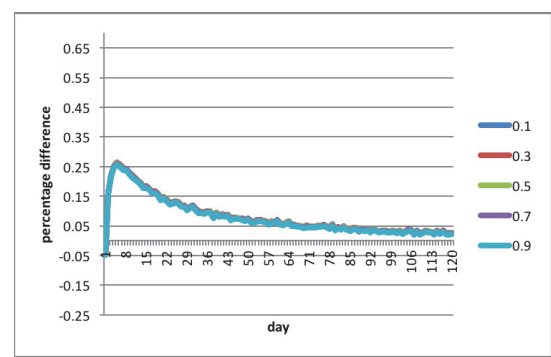

(b) Capacity $=8$

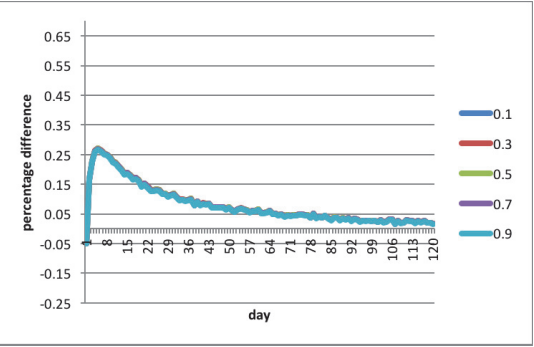

(d) Capacity $=10$

Figure 10: Average daily gaps (\%) for diversity $=15$ 


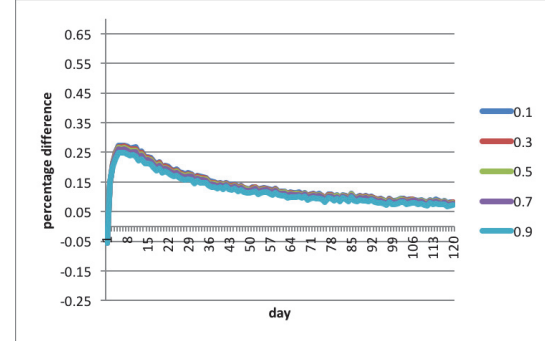

(a) Capacity $=7$

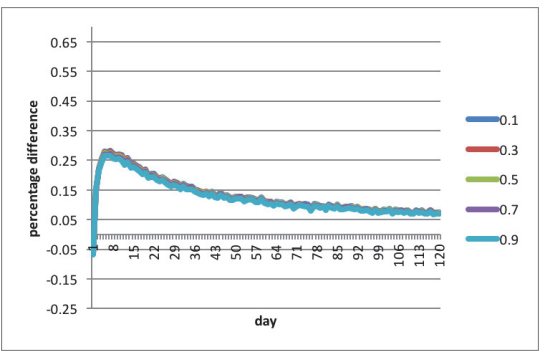

(c) Capacity $=9$

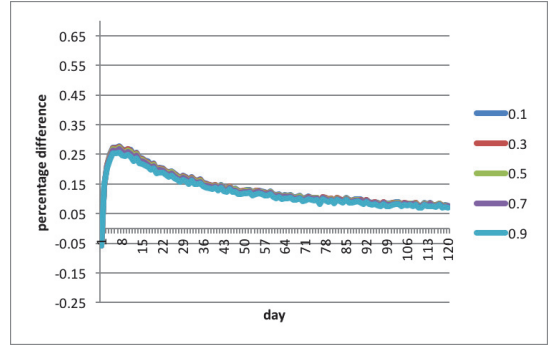

(b) Capacity $=8$

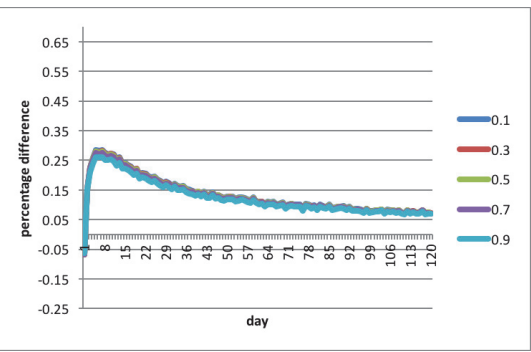

(d) Capacity $=10$

Figure 11: Average daily gaps (\%) for diversity $=20$

\section{Acknowledgements}

This material is based upon work supported by the National Science Foundation under Grant No. CMMI-1266010.

\section{References}

[1] Hays plc, The hays global skills index, available from http: //www.hays.com.mx/cs/groups/hays_common/@mx/@content/ documents/digitalasset/hays_1253572.pdf (2014).

[2] C. K. Prahalad, In volatile times, agility rules, BusinessWeek (September 21, 2009) 80.

[3] T. P. Wright, Factors affecting the cost of airplanes, Journal of Aeronautical Sciences 4 (3) (1936) 122-128. 
[4] R. W. Conway, A. Schultz, The manufacturing progress function, Dept. of Industrial and Engineering Administration, Cornell University, 1958.

[5] I. Venezia, On the statistical origins of the learning curve, European Journal of Operational Research 19 (2) (1985) 191-200.

[6] E. Cochran, New concepts of the learning curve, The Journal of Industrial Engineering 11 (4) (1960) 317-327.

[7] C.-A. E. Moulton, A. Dubrowski, H. Macrae, B. Graham, E. Grober, R. Reznick, Teaching surgical skills: what kind of practice makes perfect?: a randomized, controlled trial., Annals of surgery 244 (3) (2006) 400-9.

[8] G. Cachon, C. Terweisch, Operations Management, McGraw-Hill Education, New York, 2016.

[9] J. R. Meredith, S. M. Shafer, Operations and Supply Chain Management for MBAs, sixth Edition, John Wiley \& Sons, Hoboken, New Jersey, 2016.

[10] E. M. Dar-El, Human Learning: From Learning Curves to Learning Organizations, Vol. 29 of International Series in Operations Research \& Management Science, Kluwer Academic Publishers, Boston, 2000.

[11] M. Y. Jaber, S. Sikström, A numerical comparison of three potential learning and forgetting models, International Journal of Production Economics 92 (3) (2004) 281-294.

[12] M. Y. Jaber, Learning and Forgetting Models and Their Applications, in: A. B. Badiru (Ed.), Handbook of Industrial and Systems Engineering, Industrial Innovation Series, CRC Press, Boca Raton, FL, USA, 2006, Ch. 30, pp. $30-1-30-27$.

[13] M. J. Anzanello, F. S. Fogliatto, Learning curve models and applications: Literature review and research directions, International Journal of Industrial Ergonomics 41 (5) (2011) 573-583. 
[14] D. Nembhard, N. Osothsilp, Learning and forgetting-based worker selection for tasks of varying complexity, Journal of the Operational Research Society 56 (5) (2005) 576-587.

[15] L. Argote, Organizational Learning: Creating, Retaining, and Transferring Knowledge, Kluwer, Norwell, MA, 1999.

[16] J. A. Buzacott, The impact of worker differences on production system output, International Journal of Production Economics 78 (1) (2002) 3744.

[17] S. M. Shafer, D. A. Nembhard, M. V. Uzumeri, The effects of worker learning, forgetting, and heterogeneity on assembly line productivity, Management Science 47 (12) (2001) 1639-1653.

[18] X. Chen, B. W. Thomas, M. Hewitt, The technician routing problem with experience-based service times ?, Omega 61 (2015) 49-61.

[19] N. Gans, Y.-P. Zhou, Managing Learning and Turnover in Employee Staffing, Operations Research 50 (6) (2002) 991-1006.

[20] S. Sayin, S. Karabati, Assigning cross-trained workers to departments: A two-stage optimization model to maximize utility and skill improvement, European Journal of Operational Research 176 (3) (2007) 1643-1658.

[21] D. Biskup, Single-machine scheduling with learning considerations, European Journal Of Operational Research 188 (2008) 315-329.

[22] W. J. Gutjahr, S. Katzensteiner, P. Reiter, C. Stummer, M. Denk, Competence-driven project portfolio selection, scheduling and staff assignment, Central European Journal of Operations Research 16 (3) (2008) 281306.

[23] W. J. Gutjahr, Optimal dynamic portfolio selection for projects under a competence development model, OR Spectrum 33 (1) (2009) 173-206. 
[24] H. Zhong, R. W. Hall, M. Dessouky, Territory planning and vehicle dispatching with driver learning, Transportation Science 41 (1) (2007) 74-89.

[25] J. Fowler, P. Wirojanagud, E. Gel, Heuristics for workforce planning with worker differences, European Journal of Operational Research 190 (3) (2008) 724-740.

[26] D. a. Nembhard, F. Bentefouet, Parallel system scheduling with general worker learning and forgetting, International Journal of Production Economics 139 (2012) 533-542.

[27] A. Corominas, J. Olivella, R. Pastor, A model for the assignment of a set of tasks when work performance depends on experience of all tasks involved, International Journal of Production Economics 126 (2) (2010) 335-340.

[28] D. A. Nembhard, B. A. Norman, Cross Training in Production Systems with Human Learning and Forgetting, in: D. A. Nembhard (Ed.), Workforce Cross Training, CRC Press, Boca Raton, FL, USA, 2007, Ch. 4, pp. 111-129.

[29] C. Heimerl, R. Kolisch, Work assignment to and qualification of multiskilled human resources under knowledge depreciation and company skill level targets, International Journal of Production Research 48 (13) (2010) 3759-3781.

[30] M. Hewitt, A. Chacosky, S. E. Grasman, B. W. Thomas, Integer programming techniques for solving non-linear workforce planning models with learning, European Journal of Operational Research 242 (3) (2015) 942950.

[31] H. Jin, M. Hewitt, B. W. Thomas, Integer programming techniques for makespan models that recognize human learning, Available from http://www.researchgate. net/publication/281374510_Integer_ Programming_Techniques_for_Makespan_Models_that_Recognize_ Human_Learning (Submitted for publication). 
[32] S. Valeva, M. Hewitt, B. W. Thomas, A matheuristic for workforce planning with employee learning and stochastic demand, Available

[33] S. Valeva, M. Hewitt, B. W. Thomas, K. G. Brown, Stochastic task assignment with learning, Available from http://www.researchgate. net/publication/298556181_Stochastic_Task_Assignment_with_ Learning (Submitted for publication).

[34] P. De Bruecker, J. Van den Bergh, J. Beliën, E. Demeulemeester, Workforce planning incorporating skills: state of the art, European Journal of Operational Research 243 (1) (2015) 1-16.

[35] P.-F. Dutot, A. Laugier, A.-M. Bustos, Technicians and Interventions Scheduling for Telecommunications, Tech. rep., France Telcom Research and Development (2006).

[36] C. A. Hurkens, Incorporating the strength of MIP modeling in schedule construction, RAIRO - Operations Research 43 (04) (2009) 409-420.

[37] J.-F. Cordeau, G. Laporte, F. Pasin, S. Ropke, Scheduling technicians and tasks in a telecommunications company, Journal of Scheduling 13 (4) (2010) 393-409.

[38] H. Hashimoto, S. Boussier, M. Vasquez, C. Wilbaut, A GRASP-based approach for technicians and interventions scheduling for telecommunications, Annals of Operations Research 183 (1) (2011) 143-161.

[39] M. Frrat, C. Hurkens, An improved MIP-based approach for a multi-skill workforce scheduling problem, Journal of Scheduling 15 (3) (2012) 363-380.

[40] D. Lesaint, C. Voudouris, N. Azarmi, Dynamic Workforce Scheduling for British Telecommunications plc, Interfaces 30 (1) (2000) 45-56. 
[41] H. Qin, W. Ming, Z. Zhang, Y. Xie, A. Lim, A tabu search algorithm for the multi-period inspector scheduling problem, Computers \& Operations Research 59 (C) (2015) 78-93.

[42] G. k. Ceyhan, Ö. Özpeynirci, A branch and price algorithm for the pharmacy duty scheduling problem, Computers \& Operations Research 72 (C) (2016) 175-182.

[43] E. Zamorano, R. Stolletz, Branch-and-price approaches for the multiperiod technician routing and scheduling problem, European Journal of Operational Research 257 (1) (2017) 55-68.

[44] G. Rix, L.-M. Rousseau, G. Pesant, Solving a multi-period log-truck scheduling problem with column generation, in: 34th Council on Forest Engineering Annual Meeting. Quebec, QC, Canada, 2011.

[45] K. H. Kang, Y. H. Lee, B. K. Lee, An exact algorithm for multi depot and multi period vehicle scheduling problem, in: International Conference on Computational Science and Its Applications, Springer, 2005, pp. 350-359.

[46] J.-U. Kim, Y.-D. Kim, A decomposition approach to a multi-period vehicle scheduling problem, Omega 27 (4) (1999) 421-430.

[47] M. Babaei, M. Mohammadi, S. T. Fatemi, M. Sobhanallahi, et al., Solving multi-level, multi-product and multi-period lot sizing and scheduling problem in permutation flow shop, International Journal of Science and

[48] J. P. Vielma, A. T. Murray, D. Ryan, A. Weintraub, Improved solution techniques for multiperiod area-based harvest scheduling problems, United States Department of Agriculture Forest Service General Technical Report PNW 656 (2005) 285.

695

[49] E. Tsang, C. Voudouris, Fast local search and guided local search and their application to British Telecom's workforce scheduling problem, Operations Research Letters 20 (3) (1997) 119-127. 
[50] J. Xu, S. Y. Chiu, Effective heuristic procedures for a field technician scheduling problem, Journal of Heuristics 7 (2001) 495-509.

[51] A. Alsheddy, E. P. K. Tsang, Empowerment scheduling for a field workforce, Journal of Scheduling 14 (6) (2011) 639-654.

[52] A. Kovacs, S. Parragh, K. Doerner, R. Hartl, Adaptive large neighborhood search for service technician routing and scheduling problems, Journal of Scheduling 15 (2012) 579-600.

[53] V. Pillac, C. Gueret, A. L. Medaglia, A parallel matheuristic for the technician routing and scheduling problem, Optimization Letters 7 (7) (2013) $1525-1535$.

[54] C. E. Cortés, M. Gendreau, L. M. Rousseau, S. Souyris, A. Weintraub, Branch-and-price and constraint programming for solving a real-life technician dispatching problem, European Journal of Operational Research 238 (1) (2014) 300-312.

[55] S. Souyris, C. E. Cortés, F. Ordóñez, A. Weintraub, A robust optimization approach to dispatching technicians under stochastic service times, Optimization Letters 7 (7) (2013) 1549-1568.

[56] S. Binart, P. Dejax, M. Gendreau, F. Semet, A 2-Stage Method for a Field Service Routing Problem with Stochastic Travel and Service Times, Technical Report CIRRELT-2013-76, CIRRELT, Montreal, Canada (2013).

[57] A. Weintraub, J. Aboud, C. Fernandez, G. Laporte, E. Ramirez, An emergency vehicle dispatching system for an electric utility in Chile, Journal of Operational Research Society 44 (10) (2012) 690-696.

[58] N. Bostel, P. Dejax, P. Guez, F. Tricoire, Multiperiod Planning and Routing on a Rolling Horizon for Field Force Optimization Logistics, in: B. Golden, S. Raghavan, E. Wasil (Eds.), The Vehicle Routing Problem: Latest Advances and New Challenges, Vol. 43 of Operations Research/Computer Science Interfaces, Springer US, Boston, MA, 2008, pp. 503-525. 
[59] V. Pillac, C. Guéret, A. Medaglia, On the Dynamic Technician Routing and Scheduling Problem, Technical Report 12/5/AUTO, Ecole des Mines de Nantes, Nantes, France (2012).

[60] V. Pillac, Dynamic vehicle routing: solution methods and computational tools, Doctoral thesis, Universite Nantes Anger Le Mans and Universidad des los Andes (2012).

[61] R. W. Bent, P. Van Hentenryck, Scenario-based planning for partially dynamic vehicle routing with stochastic customers, Operations Research 52 (6) (2004) 977-987.

[62] J. Olivella, An Experiment on Task Performance Forecasting Based on the Experience of Different Tasks, in: Proceedings of I7th International Conference on Knowledge Management, Graz, Austria, 2007, pp. 305-312.

[63] J. C. Goodson, B. W. Thomas, J. W. Ohlmann, A rollout algorithm framework for heuristic solutions to finite-horizon stochastic dynamic programs, Available from http://slu.edu/ goodson/papers/ GoodsonRolloutFramework.pdf (June 2015).

[64] D. A. Nembhard, F. Bentefouet, Selection , grouping , and assignment policies with learning-by-doing and knowledge transfer, Computers \& Industrial Engineering 79 (2015) 175-187.

[65] G. Hendrickson, W. B. Schroeder, Transfer of training in learning to hit a submerged target, Journal of Educational Psychology 32 (3) (1941) 205213.

[66] F. J. Bianco, A. M. Cronin, E. A. Klein, E. E. Pontes, P. T. Scardino, A. J. Vickers, Fellowship training as a modifier of the surgical learning curve, Academic Medicine: Journal of the Association of American Medical Colleges 85 (5) (2010) 863-868.

[67] R. J. Batt, C. Terwiesch, Doctors Under Load : An Empirical Study of Service Time as a Function of Census, working paper, avail- 
able from https://opimweb.wharton.upenn.edu/files/?whdmsaction= public:main.file\&fileID=5133 (2012).

[68] L. Brown, N. Gans, A. Mandelbaum, A. Sakov, H. Shen, S. Zeltyn, L. Zhao, Statistical Analysis of a Telephone Call Center, Journal of the American Statistical Association 100 (469) (2005) 36-50. 